\title{
Afasias progresivas primarias: las afasias de lenta evolución a demencia.
}

\author{
Primary progressive aphasias: the slowly evolving aphasias to dementia. \\ Nilton Custodio $^{1,2,3}$, Sheyla Castro ${ }^{4}$, Eder Herrera-Perez ${ }^{1,5}$, David Lira ${ }^{1,2,3}$, Liza Nuñez del Prado ${ }^{6}$, Patricia \\ Cortijo $^{1,7}$, Rosa Montesinos ${ }^{1,8}$.
}

\section{RESUMEN}

La afasia progresiva primaria (APP) es un síndrome clínico neurodegenerativo caracterizado por compromiso progresivo del lenguaje, diagnosticado cuando completa tres criterios nucleares. Primero, debe existir compromiso del lenguaje, que interfiera con el uso o comprensión de las palabras. Segundo, el plan de trabajo diagnóstico debe demostrar que la enfermedad es neurodegenerativa, y además progresiva. Tercero, la afasia debe progresar relativamente aislada, sin afectación del comportamiento o de memoria episódica. El compromiso del lenguaje puede ser fluente o no fluente y puede o no interferir con la comprensión de las palabras. La memoria para hechos recientes está preservada, sin embargo los puntajes de memoria obtenidos en las pruebas mediadas verbalmente pueden ser anormales. Pueden presentarse cambios menores en personalidad o en la conducta, pero no llevan al paciente a la consulta médica y no limitan las actividades de vida diaria. Este patrón clínico es más conspicuo en los estadios iniciales de la enfermedad, y es consecuencia de la atrofia relativamente selectiva de los circuitos del lenguaje, usualmente localizado en el hemisferio izquierdo. Existen diferentes variantes clínicas de APP, cada una con un patrón característico de atrofia. Las enfermedades neuropatológicas subyacentes son heterogéneas y pueden incluir enfermedad de Alzheimer así como degeneración lobar fronto-temporal.

PALABRAS CLAVE: afasia progresiva primaria, demencia, fronto-temporal, lenguaje, variantes, semántica.

\section{SUMMARY}

Primary progressive aphasia (PPA) is a neurodegenerative clinical syndrome characterized by progressive language impairment diagnosed when three core criteria are met. First, there should be a language impairment (i.e., aphasia) that interferes with the usage or comprehension of words. Second, the neurological work-up should determine that the disease is neurodegenerative, and therefore progressive. Third, the aphasia should arise in relative isolation, without equivalent deficits of comportment or episodic memory. The language impairment can be fluent or nonfluent and may or may not interfere with word comprehension. Memory for recent events is preserved although memory scores obtained in verbally mediated tests may be abnormal. Minor changes in personality and behavior may be present but are not the leading factors that bring the patient to medical attention or that limit daily living activities. This distinctive clinical pattern is most conspicuous in the initial stages of the disease, and reflects a relatively selective atrophy of the language network, usually located in the left hemisphere. There are different

\footnotetext{
1. Unidad de Diagnóstico de Deterioro Cognitivo y Prevención de Demencia. Clínica Internacional. Lima Perú.

2. Unidad de Investigación. Clínica Internacional. Lima, Perú.

3. Servicio de Neurología. Clínica Internacional. Lima, Perú.

4. Servicio de Neurología de la Conducta. Instituto Nacional de Ciencias Neurológicas. Lima, Perú.

5. Unidad de Proyectos de Investigación. Instituto de Salud del Niño. Lima, Perú.

6. Servicio de Neurología. Clínicas Maisson de Sante. Lima, Perú.

7. Unidad de Neuropsicología. Clínica Internacional. Lima, Perú.

8. Servicio de Medicina de Rehabilitación. Clínica Internacional. Lima, Perú.
} 
clinical variants of PPA, each with a characteristic pattern of atrophy. The underlying neuropathological diseases are heterogeneous and can include Alzheimer's disease as well as frontotemporal lobar degeneration.

KEY WORDS: primary progressive aphasia, dementia, frontotemporal, language, variants, semantic.

\section{INTRODUCCION}

La degeneración lobar fronto-temporal (DLFT) es un término descriptivo macro-anatómico que refleja el relativo compromiso selectivo de los lóbulos frontal y temporal (1) y es la segunda causa más común de demencia degenerativa en el periodo pre-senil (45 a 65 años), después de la demencia de la enfermedad de Alzheimer (EA) (2,3). El espectro clínico de DLFT comprende dos síndromes claramente definidos según síntomas clínicos y el patrón regional de atrofia cerebral; el primero, caracterizado por el predominio de síntomas conductuales se denomina demencia fronto-temporal, variante conductual (DFTve) (4); y el segundo, caracterizado por el predominio del compromiso del lenguaje, se denomina afasia progresiva primaria (APP) (5).

Desde 1890 se han descrito casos de pacientes con un desorden progresivo del lenguaje que en el siglo pasado, los autores las han denominado con diversa terminología, pero los estudios sistemáticos de APP se iniciaron después que Marcel Mesulam (6) publicara en 1982 los casos de 6 pacientes con deterioro relativamente aislado y lenta progresión en el compromiso del lenguaje en su artículo titulado "Slowly progressive aphasia without generalized dementia". En el año 2004 se realizó una clasificación de las APP basada en las manifestaciones clínicas y neuropsicológicas de este grupo de patologías, siendo las tres principales variantes: La afasia progresiva primaria no fluente (APNF), la demencia semántica (DS) y la afasia progresiva logopénica (APL) $(7,8)$; sin embargo, recién en el año 2011 se describen con precisión sus criterios diagnósticos (5). Además, algunos autores describen un grupo de pacientes con alteraciones del lenguaje que no cumplen los criterios de ninguno de los grupos previamente mencionados, y a este grupo de patologías las denominan afasias progresivas no clasificables $(5,9)$. La APNF representa un $25 \%$ de los casos de DLFT y la DS un 20 a $25 \%$ $(1,7)$; mientras que la APL representaría un 5-6\% (5). Las neuroimágenes y en especial las imágenes por resonancia magnética nos permitirán observar el patrón de atrofia frontal, insular y temporal anterior que se presentan en cada una de las variantes; se debe tener en cuenta que estos cambios podrían no estar presentes en los estadios tempranos de la enfermedad donde podrían ser de gran ayuda las neuroimágenes funcionales (8).

Basados en las guías de Mesulam $(10,11)$ y GornoTempini (5), el diagnóstico de APP es posible cuando cumple los siguientes 3 criterios nucleares:

1. Desorden afásico progresivo de reciente inicio manifestado por distorsiones gradualmente intensas del uso y comprensión de la palabra que no puede ser atribuida a deficiencias motoras o perceptuales.

2. El deterioro del lenguaje es la característica neuroconductual más notoria y el impedimento principal para realizar las actividades de la vida diaria en los estadios iniciales de la enfermedad.

3. Las investigaciones diagnosticas conducen a la conclusión que la enfermedad subyacente es neurodegenerativa.

Algunos pacientes presentan signos y síntomas confinados al área del lenguaje por 10 a 14 años, mientras que otros pueden presentar en pocos años el compromiso de otras funciones cognitivas; pero, todos presentan al deterioro del lenguaje como la característica más notoria, y la que más rápido se deteriora a través del curso de la enfermedad (12). La APP se considera una forma de demencia pues causa una declinación cognitiva gradual hasta el punto que compromete las actividades de la vida diaria. También es una demencia inusual, debido a que las funciones de la memoria episódica permanecen preservadas por muchos años. Por otro lado, al contrario de las demencias amnésicas, como la enfermedad de Alzheimer (EA) quienes tienden a perder interés en actividades recreacionales y sociales, algunos pacientes con APP pueden mantener actividades de vida diaria complejas como trabajos en jardinería, carpintería y pintura.

LaAPP debe ser diferenciada de disartria progresiva pura, apraxia del lenguaje o desintegración fonológica, en donde la formación, más que el uso de la palabra, empieza a ser alterada. Debe ser diferenciada también de la EA y DFTve donde se observa una alteración para encontrar las palabras o se produce una pausa del habla, pero en quienes la característica principal radica en la alteración de la memoria (en EA) y en la conducta (en DFTvc) $(13,14)$. El inicio abrupto de afasia excluye el diagnostico de APP. Criterios de exclusión 
adicional incluyen la aparición temprana de déficit motor, amnesia, conducta anormal, agnosia asociativa o desorientación viso-espacial. Los pacientes con estas características pueden presentar los fenotipos de enfermedad de neurona motora (ENM), degeneración cortico-basal (DCB), paralisis supranuclear progresiva (PSP), EA, DFTvc, o el síndrome de atrofia cortical posterior (ACP); cada uno de los cuales puede ser acompañado por afasia progresiva, pero no primaria, por lo tanto la mera presencia de afasia no es suficiente para el diagnóstico de APP $(1,8)$.

El diagnóstico de APP es fácil, sí el paciente es examinado tempranamente, cuando los criterios nucleares pueden ser completados explícitamente. Con frecuencia los clínicos evalúan pacientes en estadios más avanzados, cuando la selectividad de la afasia ya no puede ser determinada debido a la deficiencia de comprensión del lenguaje o debido a que han surgido deficiencias en otros dominios cognitivos (14). En tales casos, una entrevista estructurada con los informantes es importante para determinar si la afasia emergió de manera aislada.

La APNF se caracteriza por compromiso de la gramática (sintaxis y morfología) pero no compromiso de la comprensión de la palabra; mientras que la DS presenta compromiso de la comprensión de palabras, pero no de la gramática; y la APL presenta dificultades intermitentes en el hallazgo de las palabras sin compromiso de la comprensión o de la gramática. La fluencia, medida en palabras por minuto, es consistentemente baja en APNF; puede no mostrar cambios o ser excesiva en DS y es altamente variable en APL. La repetición puede estar comprometida tanto en APNF y APL, pero no en DS (15).

A continuación describiremos las características más importantes de cada una las APP, tanto desde el punto de vista clínico, de neuroimágenes y patología.

\section{AFASIA PROGRESIVA PRIMARIA NO FLUENTE (APNF)}

Es conocida también como afasia no fluente progresiva, afasia progresiva primaria agramática o simplemente como la variante gramatical de las APP; se caracteriza por presentar un lenguaje hipofluente y laborioso, los pacientes se expresan con dificultad, de forma lenta, con frases cortas, simples y omiten los morfemas gramaticales (agramatismo) (5). Se considera que el lenguaje hipofluente es influenciado por algunos factores como el deterioro en el procesamiento gramátical, déficit en el planeamiento del aspecto motor del lenguaje y de alteraciones en las funciones ejecutivas. Además se presenta con parafasias fonémicas, dificultad en la comprensión de estructuras gramaticales complejas y ocasionalmente se puede observar también apraxia del habla (8), que puede ser difícil de distinguir de la disartria hipocinetica observada en pacientes con enfermedad de Parkinson. En general esta variante es la menos fluente de los tres subtipos de APP; y puede encontrarse además: acalculia, apraxia ideomotora, orofacial y alteraciones de la prosodia(15).

Los criterios de diagnóstico clínico (5) son: Agramatismo en la producción del lenguaje y/o discurso interrumpido, con esfuerzo, errores y distorsiones del sonido y 2 de los 3 siguientes:

- Afectación de la comprensión de frases gramaticalmente complejas.

- Comprensión de palabras aisladas debe estar conservada.

- Conocimiento de los objetos debe estar conservada.

Los signos extrapiramidales o el parkinsonismo leve así como bradicinecia, hipomimia y rigidez en extremidades, son hallazgos que pueden estar presentes, no están considerados dentro de los criterios diagnósticos de esta variante gramatical; sin embargo en casos dudosos del perfil lingüístico y de neuroimágenes podrían servir para la distinción con las otras variantes de las afasias progresivas primarias(16).

Las neuroimágenes muestran una atrofia con compromiso predominante a nivel fronto-insular izquierdo, compromete el giro frontal inferior, la ínsula, las áreas premotora y motora suplementaria $(5,17)$.

El examen post-morten muestra que la gran mayoría de pacientes con APP tienen patología tanto de DLFT como de EA, y se han reportado dos principales clases de alteraciones genéticas, una asociada a taupatía (DLFT-tau) y la otra con precipitados TDP43 (DLFT-TDP) (18). En la mayor parte de casos esporádicos de APNF la neuropatología es del tipo DLFT-tau; mientras que en la mayoría de los casos de DS, la neuropatología es del tipo DLFT-TDP, y el restante $20-30 \%$ de pacientes de estas dos variantes clínicas muestra la patología de EA $(18,19)$. En APL, más de la mitad de los casos tiene patología de EA; y el resto, de DLFT. Un análisis cuantitativo de casos post-morten mostraron que pacientes con diagnóstico de APP y patología EA tienen altas proporciones de ovillos neurofibrilares neocortical/entorrinal 
e izquierda/derecha que los pacientes con cuadro clínico típico de EA amnésico (20). Esta distribución atípica de degeneración neurofibrilar es compatible con la anatomía del fenotipo clínico en APP. De tal manera que determinar si un individuo con cuadro clínico de APP tiene patología de EA o DLFT es todo un reto; y evaluar el genotipo de ApoE o la evaluación metabólica a través de $\mathrm{F}^{18} \mathrm{DG}$ no ayuda en esta discriminación. De hecho el alelo $\varepsilon 4$ de ApoE, el cual es el principal factor de riesgo para patología Alzheimer en demencias amnésicas, no es un factor de riesgo para el tipo de patología Alzheimer que causa APP $(20,21)$. La evaluación de imágenes de amiloide con tomografía por emisión de positrones (TEP) y fosfotau y amiloide en líquido cefalo-raquídeo (LCR) puede ser útil para identificar pacientes con patología EA (22). Recientemente se ha descrito un desorden rápidamente progresivo del lenguaje con todas las características iniciales de APP en conjunto con enfermedad de Creutzfekdt-Jacob, sin embargo el curso tiende a ser más rápido que los casos típicos (23).

\section{AFASIA PROGRESIVA PRIMARIA VARIANTE SEMÁNTICA, o DEMENCIA SEMÁNTICA (DS)}

La DS es una entidad relativamente fácil de distinguir de las demás variantes de la DLFT y de la EA (24); se produce por una pérdida progresiva del conocimiento semántico, el paciente presenta un lenguaje fluente y gramaticalmente correcto, con un discurso vacío de significado, con circunloquios y parafasias semánticas (5); sin embargo se presenta una anomia o dificultades en la denominación por confrontación que asociada a la dificultad en la comprensión de una palabra sencilla (sobre todo de palabras de uso poco frecuente); son las características esenciales para el diagnóstico de esta variante $(1,5)$; la dificultad en la compresión de una palabra es la manifestación más evidente del déficit de memoria semántica $(1,24)$ en cambio, la comprensión de frases y la repetición están conservadas(5). Ejemplo de una conversación de un paciente con demencia semántica y su médico fue (25):

- Médico: Puede Ud. repetir la palabra "hipopótamo"

- Paciente: Si, "hipopótamo".

- Médico: Muy bien, y ¿Qué es un hipopótamo?

- Paciente: Disculpe Ud. Pero no tengo idea.

- Médico: ¿No tiene Ud. Idea de qué es?

- Paciente: Puede ser un animal grande?

- Médico: ¿Podría Ud. describir a este animal?

- Paciente: Disculpe Ud. Pero yo no puedo recordar nada acerca de esto.
Se puede presentar dislexia, disgrafia, agnosia visual asociativa, prosopagnosia, lenguaje no fluente, compromiso de la repetición y dificultades en el reconocimiento de características y usos de objetos $(5,8)$. El lenguaje espontaneo es también afectado donde se puede apreciar el uso de palabras menos específicas y muy generales como "Que", para contestar o referirse a alguna interrogante que se supone es muy clara (por ejemplo cuando se interroga por la localización geográfica de Perú en el continente americano), o referirse a "esa cosa" en lugar de utilizar palabras apropiadas (para referirse al lapicero, podría decir "esa cosa" que sirve para escribir) $(24,26)$;en estadios tardíos el paciente puede presentar cambios de comportamiento como los observados en DFTve; y la memoria episódica esta conservada, así como las habilidades viso-espaciales $(8,24)$. Otros síntomas que podrían apoyar el diagnostico de esta variante son los trastornos conductuales, como la desinhibición, comportamientos motores aberrantes y trastornos de la alimentación, observados en la DFTvc $(5,27,28)$ y dentro de los síntomas afectivos de presentación más frecuentes, tenemos irritabilidad y depresión(29).

Los criterios de diagnóstico clínico (5) incluyen: Denominación por confrontación alterada, y alteración de comprensión de palabras aisladas; y al menos 3 de los siguientes:

1. Compromiso en el conocimiento de los objetos, particularmente para términos poco utilizados o poco familiares (de baja frecuencia).

2. Dislexia superficial o disgrafia.

3. Conservación de la repetición.

4. Producción del lenguaje conservada (funciones gramatical y motora).

En las neuroimágenes se observa atrofia a predominio del lóbulo temporal anterior a predominio izquierdo que está en relación a síntomas como compulsión y falta de empatía $(5,30)$; además se puede observar compromiso parahipocampal, región entorrinal y lóbulo temporal inferior, medio y superior (17). Los hallazgos patológicos más frecuentes están en relación a cambios patológicos tipo DLFT-TDP $(5,8,30)$.

\section{AFASIA PROGRESIVA PRIMARIA LOGOPENICA (APL)}

Es un fenotipo clínico distinto a las otras variantes de las afasias progresivas primarias y de la enfermedad de Alzheimer, representando la última variante identificada de este grupo de patologías $(5,31)$; se 
caracteriza por un lenguaje marcadamente anómico, con frecuentes pausas, lo que produce un habla lenta y con una fluencia verbal falsamente reducida que podría confundirse con la variante agramatical (5).

El paciente con esta variante de afasia presenta gran dificultad en encontrar la palabra adecuada y deterioro en la repetición de oraciones y frases; no hay un franco agramatismo, pero podría presentarse parafasias fonológicas; sin embargo la prosodia y la articulación de la palabra están conservadas $(1,5)$ lo mismo que la comprensión de palabras aisladas. En este tipo de afasia los pacientes pueden señalar el objeto correcto y describir su uso cuando el evaluador lo denomina (5), ya que el trastorno parece ser secundario al daño en el circuito fonológico de la memoria de trabajo, conservando en cambio el significado de las palabras y por ello esta variante es llamada también variante fonológica (31).

Respecto a la evolución de los síntomas en APL, un estudio mostró un deterioro más rápido tanto en la fluencia verbal y la memoria episódica verbal; así como también en la atención, orientación y habilidades visuo-espaciales evolucionando a una demencia global dentro de aproximadamente 12 meses, comparado con la variante semántica que evolucionaría de forma más lenta (32). Otra característica de esta variante es la apatía, sin embargo se puede observar también irritabilidad ansiedad y agitación $(31,33)$.

Los criterios diagnósticos nucleares (5) son: compromiso en el recuerdo de palabras al discurso espontáneo y por denominación, además de compromiso en la repeticipon de frases y sentencias; $y$ al menos 3 de los siguientes:

1. Errores fonológicos en el discurso espontáneo y por denominación

2. Comprensión de palabra aislada y del conocimiento de los objetos conservados.

3. Discurso motor del habla conservado.

4. Ausencia de franco agramatismo.

Los estudios de correlación entre manifestaciones clínicas y neuroimágenes han mostrado excelente asociación (34); en las las neuroimágenes se observa atrofia a nivel de la unión parieto temporal izquierda, es decir en la región temporal posterior, parietal inferior, supramarginal y el giro angular $(24,34)$, Un estudio identificó tres variantes en relación al tiempo de enfermedad: variante típica (Afasia leve y tiempo de enfermedad de corta duración), atípica (Afasia leve y tiempo de enfermedad de larga duración) y típica severa (afasia severa y relativo tiempo de enfermedad largo); pero lo común fue que todas mostraron atrofia temporoparietal (23).

Los hallazgos neuropatológicos están en relación a los hallazgos encontrados en EA, pues más de la mitad de los casos de APL tiene las características patológicas de EA (35).

\section{DIAGNOSTICO DE AFASIA PROGRESIVA PRIMARIA}

El diagnóstico de los subtipos de las APP resulta difícil para los médicos neurólogos con poca experiencia en la evaluación neuropsicológica del lenguaje, pues está basada en las características clínicas de cada una de ellas, en los hallazgos en las neuroimágenes y en los hallazgos patológicos. Sin embargo los test neuropsicológicos (sobre todo las baterías con énfasis en la evaluación del lenguaje) podrían ser útiles y apoyar en el diagnóstico de estas patologías, ya que nos permiten identificar la presencia y el predominio de los déficits cognitivos; pero, no todos estos tests están disponibles o validados en español. Por otro lado, la dependencia estricta de las pruebas neuropsicológicas, muchas de las cuales dependen de instrucciones verbales, respuestas verbales o razonamiento verbal encubierto, puede ocasionalmente conducir a conclusiones erróneas que hay afectación de otras áreas cognitivas distintas del lenguaje. Por ejemplo los puntajes del MMSE, pueden exagerar el grado de discapacidad (36). No obstante el desorden del lenguaje en APP puede interferir en la capacidad para memorizar lista de palabras o resolver tareas de razonamiento, el paciente típicamente no tiene dificultad para recordar eventos o comportarse con buen juicio, lo que indica que la memoria explicita, razonamiento y habilidades sociales permanecen relativamente intactas. El examen neuropsicológico de un paciente con sospecha de APP tiene como objetivo demostrar la afasia, caracterizar el subtipo, e identificar los dominios cognitivos diferentes del lenguaje que están relativamente conservados. El lenguaje puede ser evaluado mediante varias baterías diseñadas para este propósito. La Western Aphasia Battery revisada (WAB-R) (37) incluye subtests que miden discurso espontaneo, comprensión de palabras y sentencias, denominación, lectura y escritura. Un cociente de afasia, derivado del WAB-R provee una medición de severidad de afasia y puede ser seguida longitudinalmente en el tiempo. La producción gramatical de sentencias puede ser evaluada con el Northwestern Anagram Test (NAT) (38), una medición 
de construcción de sentencia que no involucra la memoria de trabajo o la emisión de la voz.

El rendimiento en la evaluación de repetición puede ser evaluada con el WAB-R. Dislexia y disgrafia muy comunes en la DS pueden ser evaluadas con las palabras de excepción del Psycholinguistic Assessment of Language Processing in Aphasia (PALPA) (39). La asociación y comprensión de palabras puede ser evaluada con un subtest de 36 ítems de dificultad moderada (157-192) del Peabody Picture Vocabulary Test (PPVT-IV)(40). El Boston Naming Test (BNT) provee una medida estandarizada de denominación de objetos (41). Las habilidades no verbales deben ser evaluadas con instrumentos que disminuyan las interferencias de la afasia. Conocimiento no verbal de los objetos puede ser evaluado con el formulario de tres imágenes del Pyramids and Palm Tree test (42).

La memoria episódica puede ser evaluada con el Three Words Three Shapes (3W3S) test, prueba inicialmente diseñada para diferenciar EA de envejecimiento cognitivo saludable (43). Este test muestra que los pacientes con APP tienen un compromiso selectivo para el recuerdo de palabras pero no para las formas. La relativa preservación de las habilidades de razonamiento en APP puede ser documentada con el Visual Verbal Test, un test no verbal de flexibilidad cognitiva (44). Las funciones viso-perceptuales pueden ser evaluadas con el test de orientación de líneas (45). Los cambios conductuales, característica sobresaliente de la DFTvc, pero no presente al inicio, sí no hasta estadios avanzados de APP, pueden ser evaluados con el Frontal Behavior Inventory (46).

En todo caso, los estudios de seguimiento pueden ayudar a definir y comprender mejor las diferentes variantes de APP. Es probable que los criterios de la clasificación del consenso internacional (5) requieran algunos ajustes para acomodar aquellos casos que no reúnen los criterios de las variantes propuestas y además para prevenir la superposición de los casos que se acomodan en más de una variante. No hay lugar a dudas que las actuales guías son herramientas útiles para abordar la clasificación y el diagnóstico de APP, pero sobre todo son de gran valor para estandarizar las diversas terminologías y con ello mejorar la consistencia de los estudios de diferentes centros de investigación.

\section{CONCLUSIONES:}

1. La APP forma parte de un conjunto de entidades que afectan selectivamente los lóbulos frontales y temporales, y que junto a la variante conductual de la demencia fronto-temporal, da lugar a un témino más amplio, la degeneración lobar fronto-temporal.

2. La APP es de difícil diagnóstico, debido a que inicialmente y por muchos años, solo se evidencia compromiso de lenguaje, y tiene una lenta evolución hasta que llega a comprometer las actividades de la vida diaria.

3. La APNF se caracteriza por compromiso de la gramática, pero no de la comprensión de la palabra; mientras que la DS presenta compromiso de la comprensión de palabras, pero no de la gramática; y la APL presenta dificultades intermitentes en el hallazgo de las palabras sin compromiso de la comprensión o de la gramática.

4. En las imágenes estructurales o funcionales se debe demostrar anormalidades del área frontoinsular izquierdo en la APNF, atrofia del lóbulo temporal anterior izquierdo en DS, y anormalidades en las áreas perisilvianas posterior o parietales izquierdas en APL.

\section{Correspondencia:}

Nilton Custodio.

Unidad de Investigación. Clínica Internacional.

Garcilaso de la Vega 1420. Cercado de Lima.

Teléfono: 51-1-4319677

E-mail: niltoncustodio@neuroconsultas.com

\section{Conflictos de intereses:}

Ninguno

\section{REFERENCIAS BIBLIOGRÁFICAS}

1. Ravinovici G, Miller B. Frontotemporal lobar degeneration: Epidemiology, pathophisiology, diagnosis and management. CNS Drugs 2010;24:37598.

2. Rosso S, Donker Kaat L, Baks T, Joosse M, de Koning I, Pijnenburg Y, et al. Frontotemporal dementia in the Netherlands: patient characteristics and prevalence estimates from a population-based study. Brain 2003;126:2016-22.

3. Ratnavalli E, Brayne C, Dawson K, Hodges J. The prevalence of frontotemporal dementia. Neurology 2002;58:1615-21.

4. Rascovsky K, Hodges J, Knopman D, Mendez M, Kramer J, Neuhaus J, et al. Sensitivity of revised diagnostic criteria for the behavioural variant 
of frontotemporal dementia. Brain 2011;134:245677.

5. Gorno-Tempini M, Hillis A, Weintraub S, Kertesz A, Mendez M, Cappa S, et al. Clasification of primary progressive and its variants. Neurology 2011;76:100614.

6. Mesulam MM. Slowly progressive aphasia without generalized dementia. Ann Neurol 1982;11:592-598.

7. Gorno-Tempini ML, Dronkers NF, Rankin KP, Ogar JM, Phengrasamy L, Rosen HJ, et al. Cognition and anatomy in three variants of primary progressive aphasia. Ann. Neurol. marzo de 2004;55(3):335-46.

8. Bonner MF, Ash S, Grossman M. The new classification of primary progressive aphasia into semantic, logopenic, or nonfluent/agrammatic variants. Curr. Neurol. Neurosci. Rep. noviembre de 2010;10(6):484-90.

9. Josephs KA, Hodges JR, Snowden JS, Mackenzie IR, Neumann M, Mann DM, et al. Neuropathological background of phenotypical variability in frontotemporal dementia. Acta Neuropathol. (Berl.). agosto de 2011;122(2):137-53.

10. Mesulam MM. Primary progressive aphasia. Ann Neurol 2001;49:425- 432.

11. Mesulam MM. Primary progressive aphasia - a language-based dementia. New Engl J Med 2003;349:1535-1542.

12. Weintraub S, Rubin NP, Mesulam MM. Primary progressive aphasia. Longitudinal course, neuropsychological profile, and language features. Arch Neurol 1990;47:1329-1335.

13. Broussolle E, Bakchine S, Tommasi M, et al. Slowly progressive anarthria with late anterior opercular syndrome: a variant form of frontal cortical atrophy syndromes. J Neurol Sci 1996;144:44-58.

14. Josephs KA, Duffy JR, Strand EA, et al. Characterizing a neurodegenerative syndrome: primary progressive apraxia of speech. Brain 2012;135:1522-1536.

15. Mesulam M-M, Wieneke C, Thompson C, Rogalski E, Weintraub S. Quantitative classification of primary progressive aphasia at early and mild impairment stages. Brain 2012;135:1537-1553.

16. Kremen SA, Mendez MF, Tsai P-H, Teng E. Extrapyramidal signs in the primary progressive aphasias. Am. J. Alzheimers Dis. Other Demen. febrero de 2011;26(1):72-7.

17. Rohrer JD, Warren JD, Modat M, Ridgway GR, Douiri A, Rossor MN, et al. Patterns of cortical thinning in the language variants of frontotemporal lobar degeneration. Neurology. 5 de mayo de 2009;72(18):1562-9.

18. Mesulam M, Wicklund A, Johnson N, et al. . Alzheimer and frontotemporal pathology in subsets of primary progressive aphasia. Ann Neurol 2008;63:709-719.
19. Knibb JA, Xuereb JH, Patterson K, Hodges JR. Clinical and pathological characterization of progressive aphasia. Ann Neurol 2006;59:156-165.

20. Gefen T, Gasho K, Rademaker A, et al. Clinically concordant variations of Alzheimer patology in aphasic versus amnestic dementia. Brain 2012;135:1554-1565.

21. Rogalski E, Rademaker A, Helenewski I, et al. APOE e4 is a susceptibility factor in amnestic but not aphasic dementias. Alzheimer Dis Assoc Disord. 2011;25:159163.

22. Rabinovici GD, Jagust WJ, Furst AJ, et al. A $\beta$ amyloid and glucose metabolism in three variants of primary progressive aphasia. Ann Neurol 2008;64:388-401.

23. Mandell AM, Alexander MP, Carpenter S. CreutzfeldtJacob disease presenting as isolated aphasia. Neurology 1989;39:55-58.

24. Kertesz A, Jesso S, Harciarek M, Blair M, McMonagle P. What is semantic dementia?: a cohort study of diagnostic features and clinical boundaries. Arch. Neurol. abril de 2010;67(4):483-9.

25. Hodges JR, Patterson K. Semantic dementia: a unique clinicopathological syndrome. Lancet Neurol. noviembre de 2007;6(11):1004-14.

26. Meteyard L, Patterson K. The relation between content and structure in language production: an analysis of speech errors in semantic dementia. Brain Lang. septiembre de 2009;110(3):121-34.

27. Rosen HJ, Allison SC, Ogar JM, Amici S, Rose $\mathrm{K}$, Dronkers N, et al. Behavioral features in semantic dementia vs other forms of progressive aphasias. Neurology. 28 de noviembre de 2006;67(10):1752-6.

28. Chao SZ, Rosen HJ, Azor V, Ong H, Tse MM, Lai NB, et al. Frontotemporal dementia in eight Chinese individuals. Neurocase. 2013;19(1):76-84.

29. Thompson SA, Patterson K, Hodges JR. Left/right asymmetry of atrophy in semantic dementia: behavioral-cognitive implications. Neurology. $11 \mathrm{de}$ noviembre de 2003;61(9):1196-203.

30. Josephs KA, Hodges JR, Snowden JS, Mackenzie IR, Neumann M, Mann DM, et al. Neuropathological background of phenotypical variability in frontotemporal dementia. Acta Neuropathol. (Berl.). agosto de 2011;122(2):137-53.

31. Henry ML, Gorno-Tempini ML. The logopenic variant of primary progressive aphasia. Curr. Opin. Neurol. diciembre de 2010;23(6):633-7.

32. Leyton CE, Hsieh S, Mioshi E, Hodges JR. Cognitive decline in logopenic aphasia: more than losing words. Neurology. 5 de marzo de 2013;80(10):897-903 .

33. Rohrer JD, Warren JD. Phenomenology and anatomy of abnormal behaviours in primary progressive aphasia. J. Neurol. Sci. 15 de junio de 2010;293(12):35-8.

34. Gil-Navarro S, Lladó A, Rami L, Castellví M, Bosch B, Bargalló N, et al. Neuroimaging and biochemical 
markers in the three variants of primary progressive aphasia. Dement. Geriatr. Cogn. Disord. 2013;35(12):106-17.

35. Snowden J, Neary D, Mann D. Frontotemporal lobar degeneration: clinical and pathological relationships. Acta Neuropathol. (Berl.). julio de 2007;114(1):31-8.

36. Osher J, Wicklund A, Rademaker A, Johnson N, Weintraub S. The Mini- Mental State Examination in behavioral variant frontotemporal dementia and primary progressive aphasia. Am J Alzheimers Dis Other Demen 2007;22:468-473.

37. Kertesz A. Western Aphasia Battery- Revised (WAB-R). Austin, Texas: Pro-Ed, 2006.

38. Weintraub S, Mesulam M-M, Wieneke C, Rademaker A, Rogalski EJ, Thompson CK. The Northwestern Anagram Test: measuring sentence production in primary progressive aphasia. Am J Alzheimers Dis Other Demen 2009;24:408-416.

39. Kay JLR. Psycholinguistic Assesment of Language Processing in Aphasia. Hove: Lawrence Erlbaum Associates, 1992.

40. Dunn LA, Dunn LM. Peabody Picture Vocabulary Test-4: Pearson, 2006.
41. Kaplan E, Goodglass H, Weintraub S. The Boston Naming Test. Philadelphia: Lea \& Febiger, 1983.

42. Howard D, Patterson K. Pyramids and Palm Trees: A Test of Symantic Access From Pictures and Words. Bury St. Edmonds, Suffolk, UK: Thames Valley Test Company, 1992.

43. Weintraub S, Rogalski E, Shaw E, et al. Verbal and nonverbal memory in primary progressive aphasia: the Three Words-Three Shapes Test. Behav Neurol. 2013;26:67-76.

44. Wicklund A, Johnson N, Weintraub N. Preservation of reasoning in primary progressive aphasia: Further differentiation from Alzheimer's disease and the behavioral presentation of frontotemporal dementia. $\mathrm{J}$ Clin Exp Neuropsychol 2004;26:347-355.

45. Benton A, Hamsher K, Varney N, Speen O. Contributions to Neuropsychological Assessment, Second ed. New York: Oxford University Press, 1998.

46. Kertesz A, Nadkarni N, Davidson W, Thomas AW. The Frontal Behavioral Inventory in the differential diagnosis of frontotemporal dementia. J Int Neuropsychol Soc 2000;6:460-468. 